\title{
Theory and Phenomenology of Hybrid Mesons
}

\section{Adam P. Szczepaniak ${ }^{* \dagger}$}

Physics Department Indiana University, Bloomington, IN 47405, USA

Center for Exploration of Energy and Matter, Indiana University, Bloomington, IN 47403, USA

Jefferson Laboratory, 12000 Jefferson Avenue, Newport News, VA 23606, USA

E-mail: aszczepa@indiana.edu

This talk discusses some of latest theoretical, phenomenological and experimental results on hybrid mesons

QCD-TNT-III-From quarks and gluons to hadronic matter: A bridge too far?,

2-6 September, 2013

European Centre for Theoretical Studies in Nuclear Physics and Related Areas (ECT*), Villazzano, Trento (Italy)

\footnotetext{
${ }^{*}$ Speaker.

${ }^{\dagger}$ This work was supported in part by the U.S. Department of Energy under Grant No. DE-FG0287ER40365
} 


\section{Introduction}

Developments in particle accelerators, detection techniques and advances in theoretical and computational methods opened a new frontier in hadron physics. In recent years these have led to the tantalizing possibility that hadron spectrum may contain states that do not belong to the conventional quark model classification.

Confinement is the distinguishing feature of QCD and understanding its origins is one of the fundamental questions in physics [1]. Using lattice gauge simulation it is now not only possible to study how the confining force between color sources develops abut also make prediction for how the gluon field contributes to the hadron spectrum. In this talk I discuss the theory and phenomenology of hybrid mesons, which are expected to carry excitations of the gluon field.

\section{Hybrid mesons}

Quantum numbers of ordinary mesons are obtained by combining quantum numbers of the quark and the antiquark. For a specific quark flavor, the spin, parity and, in the case of neutral mesons, charge conduction are given by $\vec{J}=\vec{S}+\vec{L}, P=(-1)^{L+1}, C=(-1)^{L+S}$, with $L=|\vec{L}|$ being the relative orbital angular momentum and $S=|\vec{S}|=0,1$ the total quark spin. From these relations it follows that certain combinations of the $J^{P C}$ 's cannot be associated with a $q \bar{q}$ state, e.g. $J^{P C}=0^{--}, 0^{+-}, 1^{-+}, 2^{+-}, \cdots$. These are referred to as exotic. In the past they were also referred to as exotics of the second kind with the first kind reserved for flavor exotic states, e.g. isospin2 mesons to strangeness +1 baryons. In general exotics must contain other degrees of freedom besides the valance quarks. Numerous studies of exotics spectrum have been made using a variety of phenomenological models $[2,3,4,5,6,7,8,9]$, but only recently, with advances in lattice gauge simulation we have a much stronger evidence that such states might indeed exist $[10,11,12,13$, $14,15]$. It should be noted, nevertheless that hybrids, just like other ordinary $q \bar{q}$ states with masses above open decay channels become unstable resonances, and therefore are not directly accessible nether to experiment nor to numerical simulation. Various methods for extrapolation "beyond the real axis" can be used to identify a resonant state. In particular in the case of the lattice, which suffers from lack of rotational symmetry, finite volume effects, chiral extrapolation issues, etc. novel methods are at present vigorously explored [16, 17, 18, 19, 20, 21, 22]. It is important to note that even without the full machinery for mapping resonance poles of the scattering amplitude onto the finite volume lattice spectrum implemented, the existing finite volume signatures of hybrid meson are just as robust he signatures of other well established $q \bar{q}$ resonances $e . g$. the $\rho$ meson.

The lattice hadron spectrum of the isoscalar and isovector mesons (gray lines) is shown in Fig. 1 [14]. For the isoscalar states the length of the green and black lines indicates the degree of mixing between the strange and non-strange component, respectively. With the exception of states enclosed in the boxes that are discussed below, the energy levels in the left (negative parity) and the right (positive parity) columns are in a remarkable agreement with the quark model expectations. For example in the $J^{P C}=1^{--}$sector, aside the extra flavor multiplet shown in the box, the other three flavor multiplets, one at $0.9 \mathrm{GeV}$ and two at $1.8 \mathrm{GeV}$ can be classified as the ground state and the first two vector meson excitations. In the quark model, the ground state vector mesons have $L=0, S=1$ and the first excitation is either in the radial or orbital direction with $L=0$ and 


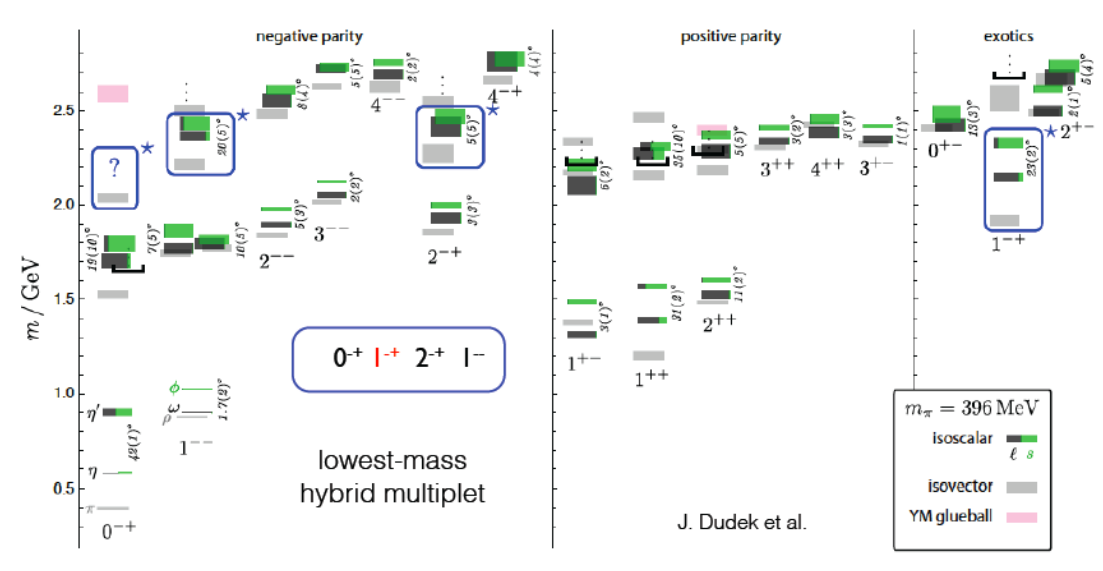

Figure 1: Light meson spectrum from LGT.

$L=2$, respectively. Considering that the lattice splitting between the ground state and the first excited level is $0.9 \mathrm{GeV}$ the second excitation region would be expected somewhere above $2.5 \mathrm{GeV}$. Instead the additional vector multiplet, which contains the states highlighted in the box, including the heaviest $s \bar{s}$ component, is found below $2.5 \mathrm{GeV}$. More importantly, lattice measurement of the wave function overlap with various quark and gluons field operators shows that while the ground and the first exited states have largest overlaps with the quark model-type operators, the boxed states overlap mainly with operators that, in addition to quark fields contain magnetic gluon fields. All these states must therefore have large gluon component in their wave functions. The right column in Fig. 1 shows states with exotic quantum numbers. These can only overlap with operators that have other fields besides those that can be reduced to the valence quark operators and obviously cannot be accounted for by the $q \bar{q}$ model. A similar pattern of $q \bar{q}$ and hybrid mesons has also been established for the lattice charmonium spectrum.

To summarize, independent of the quark mass and flavor, lattice computations indicate that there might be a multiplet of hybrid mesons located between the first and the second resonance region, which contains nearly degenerated states with $J^{P C}=0_{h}^{-+}, 1_{h}^{-+}, 2_{h}^{-+}, 1_{h}^{-+}$quantum numbers (with the subscript $h$ appended to distinguish them from the regular quark-model states), including the $1_{h}^{-+}$as the lightest exotic.

\section{Hybrids in Coulomb gauge}

The appearance of a four-state hybrid meson multiplet was predicted in $[23,24]$ in a model based on the analysis of the gluon spectrum of canonically quantized QCD in the Coulomb gauge. In a pure YM with static quarks, the Coulomb gauge calculation of the spectrum of gluon excitations gives results [25], which are consistent with lattice determination of the quark-antiquark adiabatic potentials $[26,27,28]$. In particular, as the relative separation between the quark and the antiquark is taken to zero, the excited gluon field forms a state referred to as a gluelump. Lattice determination of the gluelump spectrum shows that the lowest energy state has quantum numbers of $J_{\text {glue }}^{P C}=1^{+-}[29]$. This is quite an unexpected result, since the gauge field describes a $1^{--}$gluon. 
The appearance of the $1^{+-}$as the lowest energy state corresponds to a $1^{--}$gluon bound to the static, $q \bar{q}$ color octet in a P-wave orbital. Indeed, it can be shown that the non-abelian Coulomb potentials reverses the order of even and odd-parity orbitals, pushing the S-wave above the P-wave. The appearance of a four-state lowest multiplet of hybrids can therefore be explained. These hybrids contain the ground state constituent, color-octet $q \bar{q}$ pair and the ground state $1^{+-}$quasi-gluon. Coupling $J^{P C}$ 's of the quark pair, $J_{\text {quark }}^{P C}=0^{-+}$or $1^{--}$corresponding to zero orbital angular momentum and $S=0,1$, respectively, to the gluon $J^{P C}$ 's yields precisely the quantum numbers of lattice hybrids discussed in Sec. 2.

One can push this phenomenology further, by considering radiative transitions [11]. The multipole (low-photon energy) expansion may be an OK approximation for transitions between hybrids and the ground state or first excited $q \bar{q}$ mesons. Photon emission has both quark spin-flip and nonflip components. The former is a relativistic effect $O(1 / \mathrm{m})$ and it is suppressed for emissions from heavy quarks. Indeed, the measured radiative decay widths of the leading, $M 1$ quark-spin flip transitions between $q \bar{q}$ states, e.g. $\left.J / \psi\left(1^{--}\right) \rightarrow \gamma \eta_{c}\left(0^{-+}\right)\right)$are of the order of a few keV's, while for the non-flip $E 1$ transitions, e.g $\chi_{c 0}\left(0^{++}\right) \rightarrow \gamma J / \psi\left(1^{--}\right)$are $O(100) \mathrm{keV}$.

The quark-spin content of the non-exotic low lying hybrids, is predicted to be exactly opposite to that of the corresponding $q \bar{q}$ mesons. That is, the vector hybrid has $S=0$ and pseudo-scalar and pseudo-tensor have $S=1$ while the $q \bar{q}$ vector has $S=1$ and the other two $S=0$. Thus the order of magnitude of the magnetic dipole, $M 1$ transitions from the four hybrids (including the exotic) are expected to be larger than the $M 1$ transitions between $q \bar{q}$ states and similar to the electric dipole $E 1$ transitions between $q \bar{q}$ mesons. Ignoring hyperfine interactions the four lightest hybrids are near degenerate and have similar orbital wave functions. Thus the following decays are expected to have similar $M 1$ matrix elements [32], for $(S=1) \rightarrow \gamma(S=1): 0_{h}^{-+} \rightarrow \gamma 1^{--}, 1_{h}^{-+} \rightarrow \gamma 1^{--}$, $2_{h}^{-+} \rightarrow \gamma 1^{--}$and for $(S=0) \rightarrow \gamma(S=0), 1^{--} \rightarrow \gamma 0^{-+}$with widths, for charmonia in the range $\Gamma=10-100 \mathrm{keV}$.

\section{Experimental Status}

In recent years, BaBar, Belle, BESIII and CDF have all reported possible signatures of charmoniumlike states, which do not fail into the quark model classification [33]. One of the latest additions to the new "XYZ" resonance category is the charged chromium state, the $Z_{c}^{ \pm}(3900)$ seen in the mass spectrum of the $J / \psi \pi^{ \pm}$events from the $Y(4260) \rightarrow J \psi \pi^{+} \pi^{-}$decay [30, 31]. The $Y(4260)$ is also one of these new states and it is by now rather well established. It was discovered by BaBar [34] through initial state radiation in $e^{+} e^{-}$annihilation and confirmed by Belle [35] and CLEO [36] . There have been attempts to associate the $Y(4260)$ with a quark model state [37]. The problem is that nearby quark-model "slots" in the $4 S$ and $2 D$ multiplets are more likely to be associated with the $\psi(4415)$ and $\psi(4160)$, respectively. In fact the $Y(4260)$ is the natural candidate for the $1_{h}^{--}$ hybrid state discussed above. There is also a candidate for the $s \bar{s}$ analogue, the $Y(2175)$ seen by Belle [38]. In the light quark sector, searches have focused on hybrids with exotic quantum numbers, specifically the $1_{h}^{-+}$. A review of the experimental results can be found in [39]. The most recent sighting comes from the measurement by the COMPASS collaboration [40] of the $\eta^{\prime} \pi$ angular distribution as a function of the pair's invariant mass in the reaction $\pi^{-} p \rightarrow \eta^{\prime} \pi^{-} p$. The $\eta^{\prime} \pi$ system in the relative $\mathrm{P}$-wave has the $1^{-+}$exotic quantum numbers thus a resonance in this system 
in the mass range $1.6-2 \mathrm{GeV}$ would be a natural candidate for lightest exotic. In the past the VES collaboration [41] reported a strong intensity and phase motion characteristic to a broad resonance. The E852 experiment at BNL [42] also found a likely resonance with parameters consistent with the VES measurement. By fitting the intensity and phase of the $\eta^{\prime} \pi$ P-wave to a Breit-Wigner resonance E852 determined the mass and width of the exotic resonance to be $M=1.597_{-0.010}^{+0.045} \pm 0.010$, $\Gamma=0.340 \pm 0.50 \pm 0.04$.

\section{Summary}

This talk focused on phenomenology of hybrid hadrons. There is a growing theoretical evidence that quark-gluon hybrids should appear in the hadron spectrum and there exist experimental signatures of such states. The new experiments at the various hadron facilities are generating complicated data sets. Uncovering the mysteries of hadron spectrum demands a qualitatively new level of sophistication in analysis never before achieved. Reaction dependent amplitude are currently been developed to take full advantage of the information contained in the experimental data.

\section{References}

[1] Clay Mathematics Institute. The Millennium Prize Problems. [accessed 2012 November 22]; Available from: http://www.claymath.org/millennium/.

[2] D. Horn and J. Mandula, Phys. Rev. D, 17, 898, (1978).

[3] N. Isgur and J. E. Paton, Phys. Rev. D 31, 2910 (1985).

[4] T. Barnes, F. E. Close, F. de Viron and J. Weyers, Nucl. Phys. B 224, 241 (1983).

[5] M. S. Chanowitz and S. R. Sharpe, Nucl. Phys. B 222, 211 (1983) [Erratum-ibid. B 228, 588 (1983)].

[6] F. Buisseret and C. Semay, Phys. Rev. D 74, 114018 (2006).

[7] F. E. Close and P. R. Page, Nucl. Phys. B 443, 233 (1995).

[8] P. R. Page, E. S. Swanson and A. P. Szczepaniak, Phys. Rev. D 59, 034016 (1999).

[9] P. Guo, A. P. Szczepaniak, G. Galata, A. Vassallo and E. Santopinto, Phys. Rev. D 78, 056003 (2008).

[10] Jozef J. Dudek, Robert G. Edwards, Nilmani Mathur, and David G. Richards, Phys. Rev. D 77, 034501, (2008).

[11] Jozef J. Dudek, Robert G. Edwards, and Christopher E. Thomas, Phys. Rev. D 79, 094504, (2009).

[12] J. J. Dudek, R. G. Edwards, M. J. Peardon, D. G. Richards and C. E. Thomas, Phys. Rev. D 82, 034508 (2010).

[13] J. J. Dudek, R. G. Edwards, B. Joo, M. J. Peardon, D. G. Richards and C. E. Thomas, Phys. Rev. D 83, 111502 (2011).

[14] Jozef J. Dudek, Phys. Rev. D 84, 074023, (2011).

[15] J. J. Dudek, R. G. Edwards, P. Guo and C. E. Thomas, Phys. Rev. D 88, 094505 (2013) [Phys. Rev. D $\mathbf{8 8}, 094505$ (2013)].

[16] M. Luscher, Nucl. Phys. B 354, 531 (1991). 
[17] M. Luscher, Commun. Math. Phys. 105, 153 (1986).

[18] K. Rummukainen and S. A. Gottlieb, Nucl. Phys. B 450, 397 (1995).

[19] V. Bernard, M. Lage, U. -G. Meissner and A. Rusetsky, JHEP 1101, 019 (2011).

[20] M. T. Hansen and S. R. Sharpe, Phys. Rev. D 86, 016007 (2012).

[21] P. Guo, J. Dudek, R. Edwards and A. P. Szczepaniak, Phys. Rev. D 88, 014501 (2013).

[22] R. A. Briceno and Z. Davoudi, Phys. Rev. D 87, 094507 (2013).

[23] P. Guo, A. P. Szczepaniak, G. Galata, A. Vassallo and E. Santopinto, Phys. Rev. D 78, 056003 (2008).

[24] P. Guo, A. P. Szczepaniak, G. Galata, A. Vassallo and E. Santopinto, Phys. Rev. D 77, 056005 (2008).

[25] A. P. Szczepaniak and P. Krupinski, Phys. Rev. D 73, 116002 (2006).

[26] K. J. Juge, J. Kuti and C. J. Morningstar, Nucl. Phys. Proc. Suppl. 63, 326 (1998).

[27] K. J. Juge, J. Kuti and C. Morningstar, Phys. Rev. Lett. 90, 161601 (2003).

[28] G. S. Bali et al. [SESAM Collaboration], Phys. Rev. D 71, 114513 (2005).

[29] G. S. Bali and A. Pineda, Phys. Rev. D 69, 094001 (2004).

[30] M. Ablikim et al. [BESIII Collaboration], Phys. Rev. Lett. 110, 252001 (2013).

[31] Z. Q. Liu et al. [Belle Collaboration], Phys. Rev. Lett. 110, 252002 (2013).

[32] P. Guo, T. Yepez Martinez and A.P. Szczepaniak, in preparation

[33] J. Beringer et al. (Particle Data Group), Phys. Rev. D86, 010001 (2012).

[34] B. Aubert et al. [BABAR Collaboration], Phys. Rev. Lett. 95, 142001 (2005).

[35] C. Z. Yuan et al. [Belle Collaboration], Phys. Rev. Lett. 99, 182004 (2007).

[36] Q. He et al. [CLEO Collaboration], Phys. Rev. D 74, 091104 (2006).

[37] F.J.Llanes-Estrada, Phys. Rev. D lbf 72, 031503 (2005).

[38] C.P. Shen et al. [Belle Collaboration], Phys. Rev. D 80, 031101(R) (2009).

[39] C. A. Meyer and Y. Van Haarlem, Phys. Rev. C 82, 025208 (2010).

[40] B. Grube [COMPASS Collaboration], PoS ConfinementX , 105 (2012).

[41] G. M. Beladidze et al. [VES Collaboration], Phys. Lett. B 313, 276 (1993).

[42] E. I. Ivanov et al. [E852 Collaboration], Phys. Rev. Lett. 86, 3977 (2001). 